\title{
Validation of the "QUICK" Questionnaire-A Tool for Diagnosis of "Wearing-Off" in Patients with Parkinson's Disease
}

\author{
Pablo Martinez-Martin, MD, PhD, ${ }^{1,2} *$ Eduardo Tolosa, MD, PhD, ${ }^{3,4}$ Basilio Hernandez, MD, ${ }^{5}$ \\ and Xavier Badia, $\mathrm{MD}, \mathrm{PhD}^{6}$ on behalf of the ValidQUICK Study Group \\ ${ }^{I}$ Neuroepidemiology Unit, National Centre for Epidemiology, Carlos III, Institute of Health, Madrid, Spain \\ ${ }^{2}$ Centro de Investigación Biomédica en Red sobre Enfermedades, Neurodegenerativas (CIBERNED), Madrid, Spain \\ ${ }^{3}$ Parkinson's Disease and Movement Disorders Unit, Neurology Service, Institut, Clínic de Neurociències, Hospital Clínic de \\ Barcelona, Universitat de Barcelona, IDIBAPS, Barcelona, Spain \\ ${ }^{4}$ Centro de Investigación Biomédica en Red sobre Enfermedades, Neurodegenerativas (CIBERNED), Barcelona, Spain \\ ${ }^{5}$ Medical Department, Novartis Farmaceutica, SA, Barcelona, Spain \\ ${ }^{\sigma}$ IMS Health, Barcelona, Spain
}

\begin{abstract}
The main objective of the present study was to test the validity of a 19-items instrument (QUICK Questionnaire, QQ) as a tool for screening of WO. Two hundred twenty-two patients $(36.0 \%$ without WO; $64.0 \%$ with mild or moderate/ severe WO) were included. Diagnosis of WO by the participant neurologist was considered the "gold standard." The complete Unified Parkinson's Disease Rating Scale battery, the Cumulative Illness Rating Scale-Geriatrics, and a form about WO presence and severity were completed by the neurologist. Patients independently completed the QQ just before the clinical assessment. As a whole, patients with WO were more disabled than patients without and patients with moderate/severe WO were in worse condition than patients with mild WO. The number of QQ symptoms declared to
\end{abstract}

improve usually after the following dose of medication was $1.0 \pm 1.8$ in the group without WO, but $6.0 \pm 3.8$ in patients with WO $(P<0.001)$. A two QQ positive symptoms cut-off showed the following attributes: sensitivity, $88 \%$; specificity, $80 \%$; positive predictive value, $88.7 \%$; negative predictive value, $79 \%$; diagnostic accuracy, $85 \%$; positive and negative likelihood ratios 4.4 and 0.15 , respectively. The area under the ROC curve resulted 0.90 (CI95\%: 0.86$0.94 \%$ ). In summary, the QQ proved to be a valid screening tool to identify WO in PD patients. To our knowledge, the QQ is the only validated tool for diagnosis of WO. (C) 2008 Movement Disorder Society

Key words: Parkinson's disease; wearing-off; screening; QUICK questionnaire; diagnostic validity.
Parkinson's disease (PD) is a neurodegenerative disorder characterized by motor manifestations such as bradykinesia, tremor, rigidity, and alteration of gait and balance. A wide variety of nonmotor symptoms, such as sleep disorders, autonomic, gastrointestinal, neuropsy-

Members of the of the ValidQUICK Study Group are listed as an Appendix

*Correspondence to: Dr. P. Martinez-Martin, National Centre for Epidemiology, Carlos III Institute of Health, C/. Sinesio Delgado, 6, 28029 Madrid, Spain. E-mail: pmartinez@isciii.es

Received 5 October 2007; Revised 14 December 2007; Accepted 15 December 2007

Published online 28 February 2008 in Wiley InterScience (www. interscience.wiley.com). DOI: 10.1002/mds.21944 chological, sensory, and other kind of symptoms usually is also present. ${ }^{1-3}$

Over time, disease progression and chronic treatment with levodopa and dopamine agonists are combined to produce an array of complications (fluctuations, dyskinesias, and mental changes) which become the major problems experienced by patients. ${ }^{4-6}$

There are several modalities of fluctuations, from the gradual and predictable decline in motor performance some time (1/2 to 1 hour) before the following dose of medication (wearing-off) to the sudden and unexpected loss of mobility (sudden off). Wearing-off (WO) is easily recognized when expressed as motor fluctuation, but if manifested as nonmotor symptoms might go unnoticed. 
This is as relevant problem, because these symptoms importantly impact on patients' disability and quality of life. ${ }^{6-11}$

Stacy et al., ${ }^{10}$ developed a Patient Questionnaire which involved a number of questions on nonmotor symptoms and showed to identify WO more frequently than item 36 of the UPDRS ${ }^{12}$ and a specific Clinical Assessment Questionnaire (regarding loss of medication efficacy and presence of WO, sleepiness, dyskinesia, psychiatric complications, other dopaminergic side effects, and morning akinesia). In the same study, a 19-item short-form (Patient Card) was proposed as being potentially able to identify $100 \%$ of patients with wearing-off. ${ }^{10,13}$ An independent study with a Spanish version of this 19-items questionnaire identified more WO symptoms than a Follow-up Questionnaire and the Unified PD Rating Scale (UPDRS)- Item 36. ${ }^{14}$ However, the Patient Card was not compared with the gold-standard and the design of the study did not allow to discard a potential high proportion of false positives. Therefore, a validation study of the Patient Card Spanish version (the QUICK Questionnaire, QQ) as screening tool was proposed and is the main objective of the present study

\section{PATIENTS AND METHODS}

\section{Objectives}

To determine (1) in the setting of routine clinical practice, the quality attributes (sensitivity and specificity) of the QQ as a tool for diagnosis of WO in Spanish population with $\mathrm{PD}$; (2) the relationship between the results of QQ application and factors concerning the clinical condition and course of the disease; and (3) the relative frequency of the symptoms associated to $\mathrm{WO}$ as assessed by the QQ.

\section{Design}

Observational, multicenter, cross-sectional study.

\section{Patients}

Patients older than 30 years diagnosed as having PD (by a neurologist with competence in movement disorders) for less than 5 years prior the study and receiving pharmacological treatment for PD. Additional inclusion criteria were acceptable general health in the researcher's opinion; not taking part in any clinical trial at the time of the study; and written informed consent to participate in the study. Patients who did not match these criteria were excluded.

Sample size was calculated to allow the estimation of sensitivity and specificity for both, patients with mild WO and patients with moderate/severe WO. The follow- ing definitions were applied: (1) sensitivity was the probability that a patient with WO according the clinical criterion was classified as having this condition by means of the QQ questionnaire; and (2) specificity was the probability that a patient without WO according the clinical criterion, was classified as no experiencing WO by means of the QQ. A minimum acceptable value of $75 \%$ and a maximum possible value of $90 \%$ were hypothesized for both sensitivity and specificity. There is no general agreement about what the acceptable levels of sensitivity and specificity for an assessment test are. Acceptable levels vary depending upon the intent of the test, the setting of testing, the prevalence of the condition in the group being tested, alternate methods of assessment, and costs and benefits of testing. This range of values has been established taking into account all these factors as expected values. To demonstrate these achievements with an error $=0.25$ and statistical power $90 \%$ for sensitivity, it was estimated that 70 patients with mild WO and 70 with moderate/severe WO were needed.

For specificity at similar levels of error and power, 70 PD patients without WO should be enrolled. Therefore, at least 210 patients in three arms (no WO, mild WO, and moderate/severe WO) were included.

\section{Procedure}

The participant neurologists selected consecutive PD patients who met inclusion criteria. Patients who agree to participate in the study were asked to complete the QQ just before the clinical assessment. The neurologists had not access to these questionnaires, but were aware of the main objective of the study and were asked to record, at the time of study visit, the socio-demographic and clinical variables, with special emphasis in the presence or absence of WO.

Clinical diagnosis of WO was considered the "gold standard." WO was defined as "the generally predictable recurrence of motor and non-motor symptoms that precedes scheduled doses of antiparkinsonian medication and usually improves after those doses." 14,15 WO episodes were classified as mild when they did not interfere with performance of activities of daily living and moderate/severe when they did.

\section{Assessments}

The following instruments were applied: Socio-demographic and historical disease questionnaire; modified Hoehn and Yahr staging (HY), ${ }^{12,16}$ Schwab and England Scale (SES), ${ }^{17}$ Unified Parkinson's Disease Rating Scale (UPDRS), ${ }^{12}$ and Cumulative Illness Rating Scale-Geriatrics (CIRS-G). ${ }^{18}$ In addition, the neurologist completed a form about WO presence (yes/no) and severity if WO 
was present (mild vs. moderate/severe). At the time of the study visit and before the clinical assessment, patients independently completed the QQ.

The CIRS-G is a measure to determine the burden of chronic medical illness. It explores the severity of the medical problems in 14 organ-system categories applying a score that ranges from 0 (no problem) to 4 (extremely severe). A Severity Index is calculated on total score/total number of categories endorsed.

The QQ lists 19 symptoms related to PD: tremor, difficulty in speech, anxiety, sweating, mood changes, weakness, problems with balance, slowness, reduced dexterity, numbness, general stiffness, panic attacks, cloudy mind/dullness of thinking, abdominal discomfort, muscle cramping, difficulty getting out of the chair, experience hot and cold, pain, aching. ${ }^{10,13}$ Patients are asked to mark which of these symptoms he/she is experiencing and whether they usually improve after the next dose of medication. When any of these symptoms is reported to improve after the following dose of medication is considered a "positive response." The QQ underwent cross-cultural adaptation to ensure cultural and linguistic equivalence to the original version. ${ }^{14}$

\section{Ethical Aspects}

The protocol was reviewed and approved by the Hospital Clinic of Barcelona Research Ethics Committee. All enrolled patients signed the written informed consent to participate in the study.

\section{Data Analysis}

Descriptive statistics for the complete sample, stratified by presence or not of WO and severity of the WO episodes ( $\chi^{2}$, Student $t$-test, Mann-Whitney test).

To determine the potential diagnostic value of the QQ to detect WO and taking as gold standard the clinicians' judgment about the presence or not of WO, the following indicators were calculated: sensitivity, specificity, positive, and negative predictive values, and positive and negative likelihood ratios. A similar analysis was carried out to test the ability of the QQ to differentiate between mild and moderate/severe WO.

\section{RESULTS}

The total sample included 222 patients. According to the criterion, 80 (36.0\%) did not have WO, $71(32.0 \%)$ had mild WO, and $71(32.0 \%)$ moderate/severe WO. Patients with WO presented $2.9 \pm 1.2$ episodes/day, these episodes being significantly more frequent in patients with moderate/severe than with mild WO (3.5 vs. 2.3 , respectively; $P<0.001$ ).
Mean age of patients was $66.2 \pm 10.5$ years and $52.7 \%$ were males. Most of patients $(51.6 \%)$ had got an elementary educational level; $17.6 \%$ had not received formal education; and $11.8 \%$ had achieved university degrees. No statistical difference in age, gender, educational level, or disease duration $(4.9 \pm 2.2$ years for the complete sample) was found between patients grouped by WO presence or severity.

On the contrary, patients with WO reached a mean time from the diagnosis of PD $(3.7 \pm 1.2$ years $)$ significantly higher than patients without $(3.0 \pm 1.7)(P=$ 0.004), but no significant difference was found for WO severity categories. Similar findings were obtained when time receiving treatment for PD was considered (3.6 \pm 1.3 years with WO vs. $2.9 \pm 1.6$ without; $P=0.001$ ).

At the time of the study, $91 \%$ of patients were receiving levodopa (29.3\% with entacapone); 69.9\%, dopamine agonists; $10.8 \%$, selegiline; $7.2 \%$, rasagiline; and $1.4 \%$, amantadine. More patients with WO (97.2\%) than patients without $(80.0 \%)$ were on levodopa $(P<0.001)$, and the same was found for dopamine agonists $(76.0 \%$ vs. $58.9 \%)(P=0.007)$. None patient was on levodopa monotherapy and only $6.3 \%$ were on monotherapy with dopamine agonists, most of them (78.6\%) in the non-WO group.

Patients with WO were on a significantly more advanced disease stage than those without WO $(P<$ 0.001). Patients in HY stage 3 or 4 represented $21.3 \%$ of the WO group, whereas only $6.3 \%$ of the non-WO patients were in these stages. In addition, patients with moderate/severe WO suffered from more advanced disease than patients with mild WO $(P<0.001)$ (Table 1$)$.

SES scored significantly higher (indicating better functional ability; $P<0.001$ ) in patients without WO and in those patients with mild WO compared to those with more severe phenomena (Table 2).

Regarding the UPDRS-Mental status scores, statistically significant differences indicative of increased impairment were found between patients, both by WO presence $(P=0.024)$ and severity $(P=0.001)$. As for the WO presence, there was not significant differences between groups with respect to the UPDRS-ADL in "on" scores, but patients with moderate/severe WO were significantly more disabled $(10.4 \pm 6.0)$ than patients with mild WO $(6.2 \pm 4.8)(P<0.001)$. In the UPDRS-Motor examination section, patients experiencing WO showed major impairment than patients without WO $(28.2 \pm$ 13.9 vs. $18.5 \pm 8.9)(P<0.001)$. Again, the higher the severity of the WO symptoms, the higher the UPDRSSubscale 3 score $(P<0.001)$. The UPDRS-Complications subscale and the total UPDRS scored significantly 
TABLE 1. Distribution of patients by Hoehn and Yahr staging

\begin{tabular}{|c|c|c|c|c|c|c|c|c|c|c|}
\hline \multirow{3}{*}{$\begin{array}{l}\text { Hoehn and } \\
\text { Yahr stage }\end{array}$} & \multirow{2}{*}{\multicolumn{2}{|c|}{ Total }} & \multicolumn{4}{|c|}{ Wearing-off } & \multicolumn{4}{|c|}{ Wearing-off severity } \\
\hline & & & \multicolumn{2}{|c|}{ No* } & \multicolumn{2}{|c|}{ Yes* } & \multicolumn{2}{|c|}{ Mild* } & \multicolumn{2}{|c|}{ Mod./Sev.* } \\
\hline & $\mathrm{n}$ & $\%$ & $\mathrm{n}$ & $\%$ & $\mathrm{n}$ & $\%$ & $\mathrm{n}$ & $\%$ & $\mathrm{n}$ & $\%$ \\
\hline 1 & 30 & 13.6 & 22 & 27.5 & 8 & 5.7 & 7 & 9.9 & 1 & 1.4 \\
\hline 1.5 & 5 & 2.3 & 1 & 1.3 & 4 & 2.8 & 3 & 4.2 & 1 & 1.4 \\
\hline 2 & 108 & 48.9 & 39 & 48.8 & 69 & 48.9 & 42 & 59.2 & 27 & 38.6 \\
\hline 2.5 & 43 & 19.5 & 13 & 16.2 & 30 & 21.3 & 14 & 19.7 & 16 & 22.9 \\
\hline 3 & 33 & 14.9 & 5 & 6.3 & 28 & 19.9 & 5 & 7.0 & 23 & 32.9 \\
\hline 4 & 2 & 0.9 & 0 & 0 & 2 & 1.4 & 0 & 0 & 2 & 2.8 \\
\hline Total & 221 & 100.0 & 80 & 100.0 & 141 & 100.0 & 71 & 100.0 & 70 & 100.0 \\
\hline
\end{tabular}

*Differences between groups, $P<0.001$.

higher in patients with WO, particularly in those with moderate/severe WO (both, $P<0.001$ ) (Table 2).

The UPDRS item 36 ("off" periods predictable?) was scored 1 ("yes") by the participant neurologists in $92.1 \%$ of the patients with WO (85.9\% with mild WO; $98.5 \%$ with moderate/severe WO).

Concerning the CIRS-G, it was found that patients without WO had marginally more comorbidity than patients with WO $(P=0.04)$, but not significant differences between groups were found with regard to WO severity (Table 2).

Patients who did not experience WO declared to suffer $6.6 \pm 3.0$ symptoms of the 19 symptoms listed in the $\mathrm{QQ}$, whereas patients with WO presented $9.8 \pm 3.8(P<$ 0.001 ). On the other hand, cases with mild WO had less symptoms than those with moderate/severe $(9.0 \pm 3.7$ vs. $10.7 \pm 3.8$, respectively) $(P=0.007)$. The most frequently experienced symptoms by the complete patients sample were slowness of movement $(82 \%)$, reduced dexterity (76.6\%), and tremor (75.7\%), and the least ones were panic attacks (18\%), abdominal discomfort and hot and cold sensations $(<25 \%)$.

The number of QQ symptoms declared to improve usually after the following dose of medication was $1.0 \pm$ 1.8 in the group without WO and $6.0 \pm 3.8$ in patients with WO $(P<0.001)$ (Table 3$)$. The respective figures for mild versus moderate/severe WO were $5.4 \pm 3.3$ versus $6.7 \pm 4.0$, the difference not reaching statistical significance. Tremor (48.6\% of the sample), slowness of movement (43.7\%), and reduced dexterity (36.0\%) were the QQ symptoms most frequently improved after the following dose of medication.

In the present study, 142 patients (64\%) had WO as per the neurologist's criterion. Using a QQ 2-positive symptoms cut-off, $63.5 \%(\mathrm{n}=141)$ would be identified as patients with WO. This way, $88 \%$ of patients with genuine WO would have been detected, with a $12 \%$ proportion of false negatives and $20 \%$ of false positives. The area under the ROC curve resulted 0.90 (CI95\%: 0.86-0 0.94\%) (see Fig. 1). Taking into account the characteristics of this ROC curve, the sensitivity, specificity, and predictive values of the QQ to diagnose WO were calculated for 1 to 4 symptoms declared to improve with the following dose ("positive response"). As Table 4 shows, two positive symptoms placed all quality parameters over the reference value $75 \%$ and the agreement with the expert criterion (diagnostic accuracy) at a substantial level $(85.1 \%$; kappa value $=0.68 ; 95 \%$ CI: $0.58-0.78) .{ }^{19}$ Positive and negative likelihood ratios resulted 4.4 and 0.15 , respectively.

TABLE 2. Scores in the applied Parkinson's disease and comorbidity assessment

\begin{tabular}{|c|c|c|c|c|c|}
\hline & \multirow[b]{2}{*}{ Total $($ Mean \pm SD) } & \multicolumn{2}{|c|}{ Wearing-off (Mean \pm SD) } & \multicolumn{2}{|c|}{ Wearing-off severity (Mean $\pm \mathrm{SD})$} \\
\hline & & No & Yes & Mild & Mod./Sev. \\
\hline Schwab and England & $82.0 \pm 13.1$ & $88.8 \pm 9.5$ & $78.1 \pm 13.3^{*}$ & $83.2 \pm 11.4$ & $72.9 \pm 13.1 *$ \\
\hline UPDRS-I & $1.9 \pm 2.0$ & $1.3 \pm 1.7$ & $2.1 \pm 2.0 * *$ & $1.6 \pm 1.8$ & $2.7 \pm 2.1 * *$ \\
\hline UPDRS-II & $8.0 \pm 5.6$ & $7.0 \pm 4.8$ & $8.3 \pm 5.8$ & $6.2 \pm 4.8$ & $10.4 \pm 6.0^{*}$ \\
\hline UPDRS-III & $26.0 \pm 13.5$ & $18.5 \pm 8.9$ & $28.2 \pm 13.9^{*}$ & $22.7 \pm 9.9$ & $33.7 \pm 15.0^{*}$ \\
\hline UPDRS-IV & $3.1 \pm 2.4$ & $0.7 \pm 0.8$ & $3.9 \pm 2.2 *$ & $2.9 \pm 1.3$ & $4.9 \pm 2.5^{*}$ \\
\hline Total UPDRS & $39.5 \pm 19.3$ & $27.5 \pm 13.4$ & $43.1 \pm 19.3^{*}$ & $32.9 \pm 13.5$ & $53.3 \pm 19.0^{*}$ \\
\hline CIRS-G & $2.7 \pm 2.5$ & $3.1 \pm 2.7$ & $2.5 \pm 2.4$ & $2.6 \pm 2.4$ & $2.3 \pm 2.5$ \\
\hline
\end{tabular}

$* P<0.0001 ; * * P<0.01$

SD, standard deviation; UPDRS, Unified Parkinson's Disease Rating Scale; CIRS-G, Cumulative Illness Rating Scale-Geraitrics. 
TABLE 3. Symptoms usually improving after the following dose of medication, as per the QUICK Questionnaire

\begin{tabular}{|c|c|c|c|c|c|c|c|c|c|}
\hline & \multirow[b]{3}{*}{ QUICK Questionnaire items } & \multicolumn{4}{|c|}{ Wearing-off } & \multicolumn{4}{|c|}{ Severity } \\
\hline & & \multicolumn{2}{|c|}{ No } & \multicolumn{2}{|c|}{ Yes } & \multicolumn{2}{|c|}{ Mild } & \multicolumn{2}{|c|}{ Mod./Sev. } \\
\hline & & $\mathrm{n}$ & $\%$ & $\mathrm{n}$ & $\%$ & $\mathrm{n}$ & $\%$ & $\mathrm{n}$ & $\%$ \\
\hline 1 & Tremor & 19 & 31.7 & 89 & 82.4 & 39 & 76.5 & 50 & 87.7 \\
\hline 2 & Difficulty in speech & 5 & 23.8 & 49 & 72.1 & 19 & 65.5 & 30 & 76.9 \\
\hline 3 & Anxiety & 3 & 9.1 & 50 & 58.8 & 23 & 65.7 & 27 & 54.0 \\
\hline 4 & Experience sweating & 0 & 0 & 23 & 38.3 & 14 & 50.0 & 9 & 28.1 \\
\hline 5 & Mood changes & 8 & 22.2 & 46 & 56.1 & 22 & 55.0 & 24 & 57.1 \\
\hline 6 & Weakness & 3 & 14.3 & 50 & 67.6 & 20 & 64.5 & 30 & 69.8 \\
\hline 7 & Problems with balance & 3 & 9.1 & 52 & 55.3 & 25 & 55.6 & 27 & 55.1 \\
\hline 8 & Slowness of movement & 7 & 11.7 & 90 & 73.8 & 44 & 73.3 & 46 & 74.2 \\
\hline 9 & Reduced dexterity & 4 & 7.1 & 76 & 66.7 & 37 & 67.3 & 39 & 66.1 \\
\hline 10 & Numbness & 2 & 7.4 & 46 & 60.5 & 19 & 55.9 & 27 & 64.3 \\
\hline 11 & General stiffness & 4 & 23.5 & 57 & 74.0 & 17 & 54.8 & 40 & 87.0 \\
\hline 12 & Experience panic attacks & 1 & 12.5 & 19 & 59.4 & 6 & 46.2 & 13 & 68.4 \\
\hline 13 & Cloudy mind/Dullness thinking & 2 & 14.3 & 38 & 58.5 & 14 & 48.3 & 24 & 66.7 \\
\hline 14 & Abdominal discomfort & 1 & 8.3 & 12 & 33.3 & 6 & 42.9 & 6 & 27.3 \\
\hline 15 & Muscle cramping & 5 & 17.2 & 19 & 35.8 & 10 & 41.7 & 9 & 31.0 \\
\hline 16 & Difficulty getting out of chair & 5 & 17.9 & 54 & 70.1 & 21 & 70.0 & 33 & 70.2 \\
\hline 17 & Experience hot and cold & 1 & 8.3 & 17 & 42.5 & 9 & 37.5 & 8 & 50.0 \\
\hline 18 & Pain & 1 & 5.3 & 28 & 48.3 & 14 & 56.0 & 14 & 42.4 \\
\hline 19 & Aching & 3 & 11.5 & 38 & 52.8 & 21 & 55.3 & 17 & 50.0 \\
\hline
\end{tabular}

Boxes in gray are indicative of statistically significant difference between groups $(P<0.05)$.

The analysis demonstrated that the QQ is not useful to distinguish between mild and moderate/severe WO (area under the ROC curve $=0.59$; no one cut-off from 1 to 8 symptoms fitted the standard criteria).

\section{DISCUSSION}

The 36-item Patient Questionnaire developed by Stacy et al. ${ }^{10}$ was considered useful for detection of WO, but "too cumbersome for use during a normal office visit." 15 Therefore, these authors tried to design a shorter card

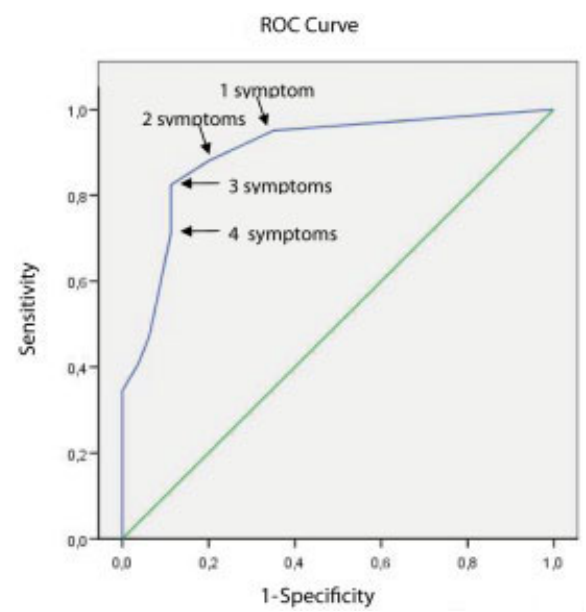

FIG. 1. The diagonal segments are caused by the connections (reference line). with enough sensitivity to be used in clinical practice and they proposed, first, the 19-item Patient Card and, later, the 9-item questionnaire. ${ }^{10,13,15,20}$ However, all these proposals have retrospectively been made by means of statistical methods applied on the original sample and no one of these versions has been validated on a target population.

The main objective of the present study was to assess the diagnostic attributes of one of these instruments, the 19-item Patient Card Spanish version (QQ), in a population of PD patients selected for this purpose. This questionnaire has been previously tested for feasibility and performance in a clinical setting, ${ }^{14}$ but never before - to our knowledge - validated as a tool for diagnosis of WO.

Sixty-four per cent of our sample population experienced WO (mean, 3 episodes/day) as judged per the clinical investigator, taken as the gold standard. No significant differences were found between patients with or without WO related to socio-demographic factors or duration of disease, but patients with WO displayed longer time since diagnosis of PD and treatment onset. Significantly more WO patients received levodopa and dopamine agonists than non-WO patients. Very few patients were on monotherapy with dopamine agonists and no one with levodopa. Therefore, relationships between presence or severity of WO and type of treatment could not be established. 
TABLE 4. Attributes of the QUICK Questionnaire for diagnosis of wearing-off

\begin{tabular}{lcccc}
\hline & $\begin{array}{c}\text { 1 or more } \\
\text { symptoms (\%) }\end{array}$ & $\begin{array}{c}\text { 2 or more } \\
\text { symptoms (\%) }\end{array}$ & $\begin{array}{c}\text { 3 or more } \\
\text { symptoms }\end{array}$ & $\begin{array}{c}\text { 4 or more } \\
\text { symptoms }\end{array}$ \\
\hline Sensitivity & 95.1 & 88.0 & 82.4 & 71.1 \\
Specificity & 65.0 & 80.0 & 88.8 & 88.8 \\
Positive predictive value & 82.8 & 88.7 & 92.9 & 91.8 \\
Negative predictive value & 88.1 & 79.0 & 74.0 & 63.4 \\
\hline
\end{tabular}

PD staging and rating scales showed, as a whole, significant increasing levels of impairment and disability from patients without WO to patients with mild and those with moderate/severe WO. This gradation was also present in relation to the number of symptoms recognized by patients in the QQ (from $6.6 \pm 3.0$ symptoms in the non-WO group to $10.7 \pm 3.8$ in patients with moderate/severe WO).

Improvement of symptoms with the following dose of medication is an outstanding characteristic of the WO fluctuation. As expected, the difference in the number of symptoms included in the QQ which patients with and without WO usually noticed to improve with the next dose was very significant. Tremor, reduced dexterity, and slowness of movement were not only the most frequently experienced symptoms for all patients but also the symptoms most frequently improved by the following dose of medication in patients with WO.

Regarding the attributes related to quality of the QQ as an instrument for detection of WO, it is relevant to highlight that the present study was carried out in the setting of the usual clinical practice and the neurologists had not access to the questionnaire once it was completed by the patient. The area under the ROC curve was indicative of a high probability for QQ values (sum of positive items) to be higher for patients suffering WO than for those who do not. The analyses of different cut-offs demonstrated that the presence of two QQ positive symptoms amply overcome the hypothesized accuracy thresholds and qualify the instrument as a reliable and useful tool for detection of WO. Sensitivity and positive predictive value (proportion of patients with WO when two QQ symptoms or more are positive) reached almost $90 \%$, a satisfactory level for screening instruments. In addition, specificity and negative predictive value (proportion of patients without WO with less than 2 positive QQ symptoms) were also satisfactory.

Previous studies demonstrated that the 32-item Patient Questionnaire $^{10}$ and the 19-item Patient Card $^{14}$ (QQ) capture the existence of WO more frequently than routine assessments. However, these studies neither allowed to exclude a potential "hypersensitivity" (high proportion of false positive) of the questionnaire (subjects with only one "positive response" were arbitrarily classified as experiencing WO) nor to determine its cut-off point against a gold-standard criterion. Data about the recently proposed 9-item version (WO Questionnaire) ${ }^{15}$ have been derived from the original study ${ }^{10}$ by means of statistical crosscheck comparison, showing again a high sensitivity, but still should be validated in a field study directed at this aim. Limitations of the study are related to this background, the "gold standard" accuracy (an anchor depending of the participant neurologists expertise), and the design of the study in terms of the established thresholds for sensitivity and specificity. Nevertheless, we believe that these limitations might not have an important influence in the validity of the present study results.

In summary, the QQ proved to be a valid screening tool to identify WO in PD patients. The findings reported here support the use of the QQ in the setting of outpatient movement disorder or general neurologist clinics, since it may facilitate the detection of WO and the identification of non motor symptoms involved in these fluctuations. Acknowledgment by the patients in the questionnaire that two symptoms usually improved or disappeared after the following medication dose provided the best performance for the QQ, with sensitivity, specificity, and predictive values higher than $75 \%$ and a global diagnostic accuracy of $85 \%$. To our knowledge, the QQ is the only validated tool in the literature for diagnosis of WO.

\section{APPENDIX}

The ValidQUICK Study Group: Arbelo JM (Hospital Insular de Gran Canaria, Gran Canaria); Arcaya J (Hospital Universitario de Salamanca, Salamanca); Bayes A (Clínica Teknon, Barcelona), Campos V (Hospital Clínico de Málaga, Málaga); Catalán MJ (Complejo Universitario San Carlos, Madrid); Durán C (Hospital Infanta Cristina, Badajoz); Escamilla F (Hospital Virgen de las Nieves, Granada); Fernández JM (Hospital de Basurto, Bilbao); García Ruiz P (Fundación Jiménez Díaz, Madrid); Genís D (Hospital Josep Trueta, Gerona); Leiva C (Hospital General, Alicante); Lezcano E (Hospital de Cruces, Vizcaya); Martí MJ (Hospital Clinic de Barcelona, Barcelona); Martínez, JC (Hospital Ramón y Cajal, 
Madrid); Mínguez A (Hospital Virgen de las Nieves, Granada); Morsi O (Hospital University of Virgen Arrixaca, Murcia); Ponce MA (Hospital Río Carrión, Palencia); Rodríguez N (Hospital de Jerez, Jerez de la Frontera); Suárez JA (Hospital Dr Negrín, Tenerife); Viguera J (Hospital Vírgen Macarena, Sevilla); Vivancos F (Hospital Universitario La Paz, Madrid).

Acknowledgments: This study was undertaken with the unrestricted support of Novartis Farmaceutica, Spain.

\section{REFERENCES}

1. Chaudhuri KR, Yates L, Martinez-Martin P. The non-motor symptom complex of Parkinson's disease: a comprehensive assessment is essential. Curr Neurol Neurosci Reports 2005;5:275-283.

2. Chaudhuri KR, Healy DG, Schapira AHV. Non-motor symptoms of Parkinson's disease: diagnosis and management. Lancet Neurol 2006;5:235-245.

3. Martinez-Martin P, Schapira AHV, Stocchi F, et al. Prevalence of non motor symptoms in Parkinson's disease in an international setting: study using non-motor symptoms questionnaire in 545 patients. Mov Disord 2007;22:1623-1629.

4. Kostic VS, Marinkovic J, Svetel M, Stefanova E, Przedborski S. The effect of stage of Parkinson's disease at the onset of levodopa therapy on development of motor complications. Eur J Neurol 2002;9:9-14.

5. Goetz CG. Parkinson's disease. Medlink neurology. San Diego: Medlink Corporation; 2005. (Available at: www.medlink.com. Accessed on July 16, 2007)

6. Witjas T, Kaphan E, Azulay JP, et al. Nonmotor fluctuations in Parkinson's disease: frequent and disabling. Neurology 2002;59: $408-413$.

7. Adler CH. Relevance of motor complications in Parkinson's disease. Neurology 2002;58(Suppl 1):51-56.

8. Raudino F. Non motor off in Parkinson's disease. Acta Neurol Scand 2001;104:312-315.
9. Weintraub D, Moberg PJ, Duda JE, Katz IR, Stern MB. Effect of psychiatric and other nonmotor symptoms on disability in Parkinson's disease. J Am Geriatr Soc 2004;52:784-788.

10. Stacy M, Bowron A, Guttman M, et al. Identification of motor and nonmotor wearing-off in Parkinson's disease: comparison of a patient questionnaire versus a clinician assessment. Mov Disord 2005;20:726-733.

11. Chaudhuri KR, Martinez-Martin P, Brown RG, et al. The metric properties of a novel non-motor symptoms scale for Parkinson's disease: results from an international pilot study. Mov Disord 2007;22:1901-1911.

12. Fahn S, Elton RL. Members of the UPDRS Development Committee. Unified Parkinson's disease rating scale. In: Fahn S, Marsden CD, Calne DB, Goldstein M, editors. Recent developments in Parkinson's diasease, Vol 2. Florham Park, NJ: Macmillan Healthcare Information; 1987. p 153-164.

13. Stacy M, The EODWO Group. Wearing-Off in Parkinson's Disease: a patient survey vs. programmed clinical evaluation. Mov Disord 2003;18:1094.

14. Martinez-Martin P, Tolosa E, Hernandez B, Badia X. The patient card questionnaire to identify wearing-off in Parkinson disease. Clin Neuropharmacol 2007;30:266-275.

15. Stacy M, Hauser R, Oertel W, et al. End-of-dose wearing off in Parkinson disease: a 9-question survey assessment. Clin Neuropharmacol 2006;29:312-321.

16. Hoehn MM, Yahr MD. Parkinsonism: onset, progression and mortality. Neurology 1967;17:427-442.

17. Schwab RS, England AC. Projection technique for evaluating surgery in Parkinson's disease. In: Gillingham FJ, Donaldson IML, editors. Third symposium on Parkinson's disease. Edinburgh: E and S Livingstone; 1969. p 152-157.

18. Miller MD, Paradis CF, Houck PR, et al. Rating chronic medical illness burden in geropsychiatric practice and research: application of the cumulative illness rating scale. Psychiatry Res 1992;41:237248.

19. Landis JR, Koch GG. The measurement of observer agreement for categorical data. Biometrics 1977;33:159-174.

20. Stacy M, Hauser R. Development of a patient questionnaire to facilitate recognition of motor and non-motor wearing-off in Parkinson's disease. J Neural Transm 2007;114:211-217. 